\title{
A Genetic Algorithm Based Approach to Service Identification
}

\author{
Ali Kazemi ${ }^{1}$, Ali Rostampour ${ }^{1}$, Pooyan Jamshidi ${ }^{2}$, Eslam Nazemi ${ }^{1}$, Fereidoon Shams ${ }^{1}$, Ali Nasirzadeh Azizkandi \\ ${ }^{1}$ Automated Software Engineering Research Group \\ Faculty of Electrical and Computer Engineering, \\ Shahid Beheshti University, Tehran, Iran \\ \{ali.kazemi, a.rostampour\}@mail.sbu.ac.ir, \{nazemi, f_shams\}@sbu.ac.ir, ali_nasirzadeh@tabrizu.ac.ir \\ ${ }^{2}$ Lero - The Irish Software Engineering Research Centre, \\ School of Computing, Dublin City University, Dublin, Ireland \\ pooyan.jamshidi@computing.dcu.ie
}

\begin{abstract}
One of the key activities in service-oriented solution development is the identification of services according to a set of predefined design principles. Existing service identification approaches are often prescriptive and based on the architect's experience, therefore might lead to non-optimal designs which results in lower performance, reduced scalability, and complicated dependencies between services. In this paper, an automated method for identifying business services has been proposed by adopting design metrics based on top-down decomposition of processes. This method takes a set of enterprise business processes as input and produces a set of non-dominated solutions representing appropriate business services using a multi-objective genetic algorithm. The method has been realized in form of a tool implementation and a case study has been conducted to show its applicability.
\end{abstract}

Keywords- Service Identification; Automated Software Engineering; Genetic Algorithm.

\section{INTRODUCTION}

Due to rapid changes in business environments, organizations face various challenges in order to adapt themselves with this turbulent environment. ServiceOriented Computing (SOC) is one of the emerging approaches to build adaptable organizational systems [1] Service identification is the first phase of designing such adaptable systems that have key role in their life cycle [2] The goal of this phase is producing a set of candidate services for constructing the target solution [1], [2], [3].There are lots of research aiming to develop methods that produce high quality services; however, they suffer from serious shortcomings [1], [4]. The inadequacies related to previous approaches are summarized as follows:

- Lack of a comprehensive and automated approach for identifying enterprise-level services.

- Subjectivity of the identified services to the experience of architect.

- Lack of utilizing standard inputs such as BPMN or activity diagrams.

In this paper, a novel identification method is proposed that aims to address above issues by supporting capabilities of automation, adopting technical metrics, and utilizing enterprise-level artifacts. We assume that the enterprise has processes which have been properly modeled using a modeling language like BPMN. Thus this enterprise can shift to service-orientation just when it is in a proper state from business process point of view.

The proposed method includes three phases: design metric definition, service sets identification and selection of the most appropriate services which satisfies the goals of business domains. A set of goals and business processes are taken as inputs and a set of services which properly support business processes are produced as outputs. Using this approach, the main focus is drawn to the metrics and consequently some advantages are resulted such as increasing the quality of solution, improving the role of the architect by automating tasks and also satisfaction of requirements and goals of business by identifying appropriate services.

The rest of this paper is organized as follows. In section II, the most related work is briefly reviewed. In section III, the basic concepts of business process and multi-objective optimization are defined. In Section IV, the service identification problem is introduced and formally defined. A novel method in order to solve the service identification problem is proposed in section $\mathrm{V}$. Then, it has been detailed in section VI by solving the problem with genetic algorithm (GA). The case study has been conducted in section VII. Finally, the conclusion and further research directions are discussed in sections VIII.

\section{RELATED WORK}

In this section, we briefly review the most related work in service identification.

\section{A. Non-Automated Service Identification Methods}

Arsanjani et al. [5] proposed an end-to-end software development method to produce service-oriented solutions called SOMA. One of the phases in life cycle of this methodology is service identification. Service identification follows three main complementary techniques: Goal-service modeling, domain decomposition and existing asset analysis. These complementary techniques prepare prescriptive tasks and guidelines that can be used as best practices in real projects. Unfortunately, the quality of the identified services depends on experience and knowledge of the user who uses these guidelines.

In [6], the main objective is the identification of enterprise-level services, however some aspects of service identification methodology like the utilization of tools has 
been ignored. A top-down and bottom-up technique for service identification has proposed in this methodology. Although the proposed method can be used in enterprise scale, it has not used quantitative metrics for identifying service with a good level of abstraction.

Qian et al. [7] have proposed a method for service identification using business process decomposition. The architect in this method identifies various service schemas based on personal experience, and then using design metrics measures their quality attributes. Also a particular tool has been developed so that the architect can monitor metric values and analyze appropriateness of services while identifying services.

Jamshidi et al. [8] have proposed a process for identifying and specifying service-oriented architectural elements at the enterprise level from business models. They assert that service identification is a multi-objective optimization problem. Although they claim that their proposed process has the automation capability based on model-driven principles and emphasis on adopting technical metrics, they could not delineate the problem formally and propose an automated method based on quantitative service design principles.

Prescriptive methods suffer from various issues including dependency on the architect's experience, lack of support for automation such as tools which help architects to identify services.

\section{B. Semi-Automated Service Identification Methods}

Jain et al. [9] proposed a method, called MOGA-WSI, to identify web services. This method which was the first effort to automatic web service identification takes an analysis model as an input and derives candidate web services. In this method, classes in the object model are grouped into appropriate web services based on static and dynamic relationship between them. A primary grouping of classes is constructed using the Maximum Spanning Tree algorithm and then the optimized solution is achieved using GA. Unfortunately, determining the total strength of the relationship between two classes required a weighted average of human judgments on several dimensions of relationships among the classes.

According to Moody and Flitman [10], this was an enormous task and defeated the purpose of automation. Although this method tries to be automated, its input model (i.e. analysis model) is not easily available at the right time. Referring to the service-oriented solution lifecycle [5], only business level models are available to service modeling activities. However, in order to utilize this method in the lifecycle, we should take into account the efforts and overhead for constructing the analysis model and this makes the MOGA-WSI less efficient and less practical. Moreover, in this method, no solution on output service set selection is provided and this task is merely relies on the architect's expertise. Thus approaches that use enterprise-level assets (e.g. processes) and then identify appropriate services according to enterprise business goals are more desired.

Azevedo et al in [11] have presented a systematic method for service identification in three phases which are:
"Selection of Activities", "Identification and Classification of Candidate Services" and "Consolidation of Candidate Services". In the first phase, a set of activity is taken as input and then those activities which can be done automatically are selected. In the second phase a set of heuristic has been defined which are applied on the activities selected from first phase. The outputs of this phase are candidate services which are obtained by syntactical and semantic analysis of the process model and making use of mentioned set of predefined heuristics. In the third phase, the obtained services are consolidated. Although in this paper a systematic approach has been proposed for service identification, most of proposed heuristics cannot be measured automatically. For example one of the heuristics is that the candidate services must be identified from business rules. But this is a general guideline and there is not any explanation about how to do it and especially the way of its automation while this is an enormous and comparatively complex task. Also this paper has not used any technical metric to ensure appropriateness of service abstraction level.

Strosnider el al. [12] have introduced an approach namely model-driven business transformation (MDBT), which uses a model-driven software synthesis technology to (semi-) automatically generate production-quality business service component from high-level business process model. They present the business entity life cycle analysis (BELA) technique for MDBT-based SOA solution realization and its integration into SOMA framework. Although they have implemented a tool to semi-automate the generation of services which lead to reduce production costs and time, a few deficiencies are yet to be addressed. Since the proposed approach focuses on entities rather than activities in order to model the behavior of the target solution, behaviors which cannot be inferred based on entities interaction, would be missed.

Previous service identification methods are often dependent on the architect's experience and result in nonoptimal designs since they do not use quantitative metrics. Limited support of automation is another deficiency of previous methods.

\section{BASIC CONCEPTS}

In this section, the relevant terminologies and its implications are defined.

Definition 1 (Business process): A business process (BP) is a set of logically related activities which are designed to achieve pre-defined business goals [13].

Definition 2 (Business Activity): A Business Activity is an atomic task in a business process [13].

Definition 3 (Data Flow): Defines the interaction between activities, for example the outputs of an activity may be used as inputs of other activity [7]. Each data flow contains one or some business entities which are exchanged as a message between activities.

Definition 4 (Business Entity): A Business Entity (BE) is a dominant data unit. Each $\mathrm{BE}$ has a relative complexity so that the designer can estimate it base on his/her experience [7]. 
Definition 5 (Multi-Objective optimization): A MultiObjective optimization problem is defined as follows [14]:

$$
\begin{gathered}
\min f(x)=\left\{f_{1}(x), f_{2}(x), \ldots, f_{M}(x)\right\} \\
x \in X^{n_{x}} \\
\text { s.t. } g(x) \geq 0, h \geq(x)=0
\end{gathered}
$$

Where $\mathrm{x}$ is the vector of decision variables bounded by the decision space, $\mathrm{X}^{\mathrm{n}_{\mathrm{x}}}$ and $\mathrm{f}$ are the set of objectives to be minimized.

Definition 6 (Pareto optimality): A solution $x \in \Omega$ is said to be Pareto Optimal with respect to (w.r.t.) $\Omega$ if and only if (iff) there is no $\mathrm{x} \in \Omega$ for which $\mathrm{v}=\mathrm{F}(\hat{\mathrm{x}})=\left(\mathrm{f}_{1}(\hat{\mathrm{x}}), \ldots, \mathrm{f}_{\mathrm{k}}(\hat{\mathrm{x}})\right) \quad$ dominates $\mathrm{u}=\mathrm{F}(\mathrm{x})=$ $\left(f_{1}(x), \ldots, f_{k}(x)\right)[14]$. In other words a solution is in Pareto optimal set if there is no solution which dominates it.

Definition 7 (Pareto Dominance): A vector $\mathrm{u}=$ $\left.\left(\mathrm{u}_{1}, \ldots, \mathrm{u}_{\mathrm{k}}\right)\right)$ is said to dominate another vector $v=$ $\left(v_{1}, \ldots, v_{k}\right.$ ) (denotedbyu $\leqslant v$ if and only if $\mathrm{u}$ is partially less than v, i.e, $\forall \mathrm{i} \in\{1, \ldots, \mathrm{k}\}, \mathrm{u}_{\mathrm{i}} \leq \mathrm{v}_{\mathrm{i}} \wedge \exists \mathrm{i} \in\{1, \ldots, \mathrm{k}\}: \mathrm{u}_{\mathrm{i}}<$ $\mathrm{v}_{\mathrm{i}}$ [14].

Definition 8 (Pareto-Optimal Set): Pareto optimal set is defined like:

$$
\mathrm{P}^{*}=\{\mathrm{x} \in \Omega / \nexists \dot{\mathrm{x}} \in \Omega \mathrm{F}(\mathrm{x}) \preccurlyeq \mathrm{F}(\mathrm{x})\}[14] .
$$

This means that a Pareto solution set is a set of solutions which are not dominated by any solution.

\section{SERVICE IDENTIFICATION PROBLEM ANALYSIS}

In this section, the service identification problem is introduced and formally defined.

\section{A. Descriptive problem delineation}

Identifying key abstractions (i.e. architectural, analysis, design elements) is considered as one of the difficult tasks in classical software development [15]. Levi and Arsanjani [16] claim that "design engineers often have difficulty determining the initial set of key abstractions that constitute the key elements of a domain in a repeatable and nonarbitrary fashion." The identification of key abstractions is most likely one of the first activities in the modeling of an enterprise solution and therefore, errors made during this step can flow down through downstream (detailed design and implementation) activities [17]. Therefore, effectiveness (identifying the right elements) and efficiency (identifying the elements in productive manner) are the most important factors in the initial steps of enterprise solution lifecycles.

In service-oriented analysis and design methods (such as SOMA [4]), identifying key abstractions (i.e. services) is followed by their specification and realization. Although some might argue that object-oriented analysis and design (OOAD) techniques can be used as a good starting point for services, their main emphasis are on micro-level abstractions. Services, on the other hand, are businessaligned entities and therefore, are at a much higher level of abstraction than are objects and components [18]. Hence, there needs to be a higher level of modeling and design principles that deal with the first-class constructs of a SOA. This is an area where SOA introduced innovative concepts and ideas that were not addressed by other paradigms. Although applying valid prescriptive guidance in service identification gives us the right services, it is important to achieve it in a productive manner. Therefore, in the present paper, we put forward an automated method for service identification.

Referring to the SOMA [4], different approaches can be adopted to identify services, namely top-down (domain decomposition), bottom-up (existing system analysis) and meet-in-the-middle (goal service modeling). The top-down approach is about decomposing business elements and refining them into a granularity that makes sense for services. The bottom-up approach is about analyzing existing IT assets and finding functionality that could be exposed as services, to be reused by many. The last one is about reconciling needs and what is already provided by existing IT assets. Since the input artifacts to top-down service identification are business side and non-technical, by adopting technical metrics, effective, business aligned, and model-driven consistent services could be derived. On the other hand, the other approaches take a look at the existing application portfolio and other assets and standards that may be not be ready for improvement, not business aligned, and not consistent with model-driven principles. Therefore, in the present work, we begin with enterprise business model and derive the effective service set based on the information provided only by that model.

Based on these premises, the service identification problem, for which we want to propose an automated method, could be formulated as follows: "How good service abstractions (at the right level of granularity) with acceptable technical metrics can be derived automatically from highlevel business requirements and process models?"

\section{B. Formal Delineation of the Problem}

Formally, the problem of service identification can be shown as a graph. To do so business processes (Definition 1) are used. Suppose $M=(B A, D F)$ be a directed graph so that $\mathrm{BA}$ is the set of nodes and DF is the set of its edges. In this definition nodes are correspondent to business activities (Definition 2). Moreover, there is an edge from node $i$ to node $\mathrm{j}$ if there be a data flow (Definition 3 ) between activity $\mathrm{i}$ and activity $\mathrm{j}$. Therefore, the service identification problem can be defined as partitioning of the mentioned graph. The graph must be partitioned in way that identified services (each partition of graph is correspondent to a service) be optimal from quality attributes point of view. To formulate this problem (graph partitioning), it should be delineated by multi-objective optimization models (Definition 5). Since the formulated multi-objective problem is NP-complete, we have adopted a GA algorithm to solve the problem. In this definition, objective vector is quality attributes of services which must satisfy business goals. A solution for this problem (a possible partition which is correspondent to a service set) is called Pareto (Definition 6) if there is not any other solution whose objective vector is dominant to objective vector of the Pareto solution. An objective vector $U$ dominates an objective vector $\mathrm{V}$ (Definition 7) if every entry of vector $U$ is better than every entry of vector $V$. The set of 
all Pareto solutions is called Pareto optimal set (Definition 8).

Taking the above definition into account, an example of the graph (eight activities and data flow interaction between them) is shown in Figure 1.

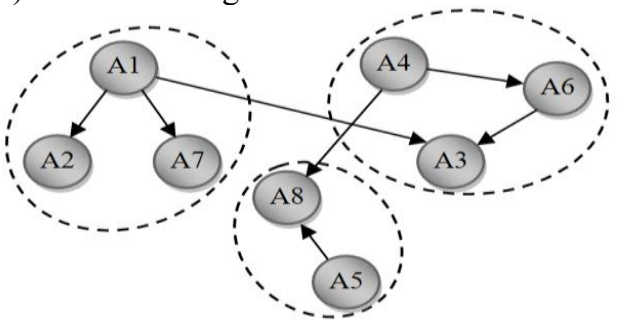

Figure 1. A sample of the graph representing a business process

Figure 1 shows a possible partitioning of the above graph and three services have been identified. These services should be optimal with respect to quality attributes such as coupling, cohesion and granularity that are defined quantitatively.

\section{THE PROPOSED METHOD}

The purpose of identifying services is to create a set of candidate services and operations associated to them [2]. Service modeling is one of the most common discussions of information technology executives and architects, and it is going to be one of the SOA challenges. Typical challenges are as follows [19]:

Challenge 1: How do we establish criteria for measuring quality attributes like granularity and reusability?

Challenge 2: How do we identify appropriate candidate services?

Challenge 3: How do we prioritize services?

In this paper we will give answers to the three aforementioned major challenges in service-oriented modeling phase. We will define a set of step-by-step automated process to give an answer to the raised challenges.

In the following we will explain the proposed service identification method. The Figure 2 shows the method in a format of a process model. This process model includes three phases which are described later in this section and deal with the mentioned challenges in service-oriented modeling and contains a set of tasks which clarify them respectively.

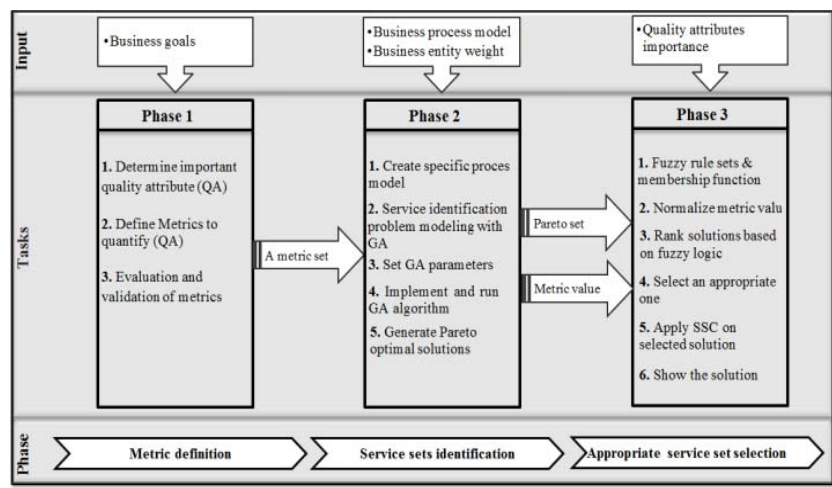

Figure 2. The proposed method

\section{A. Metric Definition Phase}

In this phase, a set of business goals are taken and set of appropriate metrics are defined or selected. Thus achieving a set of appropriate metrics from business goals is the major purpose of this phase.

First, a set of important quality attributes which cover business goals are selected. Since different quality attributes satisfy different business goals, it is required that the designer selects a set of appropriate quality attributes aligned with those goals. Normally a service-oriented system requires satisfaction of many quality attributes requirements to achieve enterprise business goals. For example, some of enterprise goals can be listed as follows [19]:

- Be agile to adapt quickly to new opportunities and potential competitive threats

- Be first to market with innovative services for its customers

- Provide streamlined business processes to reduce operating costs

Specific business goals propagate their required quality attributes, for example agility and first to market business goals mean that Adaptability, Interoperability, Scalability, and Extensibility quality attributes are the major drivers for application architecture and service development, and it is possible that the importance of these attributes differ according to business goals [21].

In the next step, appropriate metrics are defined for each quality attribute so that they can be measured automatically.

The last step of this phase is evaluation and validation of the defined metrics to make sure that those metrics are completely effective for measuring different quality attributes of services. The key output of this phase is a set of metrics which are used in the second phase.

The first phase of the proposed method deals with the first challenge in service-oriented modeling and contains a set of tasks which answer this challenge clearly.

\section{B. Service Set Identification Phase}

The ultimate goal of this phase is automatic identification of candidate service set. Business process models together with business entities and also the defined metrics in the first phase constitute the inputs of this phase. In this phase, characteristics required for the modeling of the problem according to definition 5 are derived from business process model; these characteristics themselves constitute a specific process model. Then the problem of identifying services is modeled as a genetic based multi-objective optimization algorithm. By setting the parameters of GA, implementation is done and then executed on a specific model. In an evolutionary process, this algorithm will produce a set of non-dominated solutions as a Pareto set. The key output of this phase is a Pareto set of solutions together with value of metrics related to each solution.

The second phase of the proposed method deals with the second challenge in service-oriented modeling and contains a set of tasks which answer to this challenge clearly. 


\section{Appropriate Service Set Selection Phase}

Different quality attributes in enterprise have different degrees of importance with respect to its business goals, therefore it is required that the importance of quality attributes to be specified. Therefore, the importance degree of quality attributes is one of the inputs of this phase. The other input of this phase is the Pareto solution set which is the output of the second phase.

In this phase, first, according to importance of quality attributes, a set of fuzzy rules together with their membership functions are defined. Then the metric values are normalized in a way that they are placed in 0 and 1 intervals. Ranking of Pareto solutions is the next activity which is done right after fuzzy logic, so that the Pareto solution which is mostly related to the business goals will have the highest rank. The Pareto solution with the highest rank will be transformed into a service set by SSC algorithm and finally will be showed. The key output of this phase is an optimized service set which can be used to develop service-oriented systems.

The third phase of the proposed method deals with the third challenge in service-oriented modeling and contains a set of tasks which answer to this challenge clearly.

\section{Modeling the SERVice IdentificAtion Problem WITH GA}

As stated before, the major focus of this paper is on the realization of the second phase of the proposed method.

Metric set, business process and weights corresponding to business entities will be entered in the second phase. Business process should be transformed to a process model which is suitable to our proposed method. This process model is defined as a tuple $\mathrm{M}=(\mathrm{BA}, \mathrm{DF})$, where $\mathrm{BA}$ is a set of business activities and DF is dataflow. Interaction between activities is defined based on dataflow between them which contains a set of BEs. Modeling service identification problem with GA includes chromosomes representation, assigning fitness function and defining crossover and mutation operators. By setting GA parameters like initial population, ending condition, cross-over rate and mutation probability in an evolutionary process value of metrics and Pareto set is produced as output. We will focus on details of how to model service identification problem with GA in the following subsections:

\section{- Representation and Initial Population Creation}

In order to apply the GA to a particular problem, we need to select an internal string representation for the solution space. The choice of this component is one of the critical aspects to the success or failure of the GA regarding the problem of interest. In our method, we represent each chromosome as a sequence of service activity.

Generally a business process can be partitioned at most to $n$ service, where $n$ is the number of existing activities in business process. In other words, activities in a business process can be placed in one service, two services, three services, and or $\mathrm{n}$ separate services. Each chromosome which is representative for a service set contains $\mathrm{n}$ genes. Activities inside genes are placed in at most $\mathrm{n}$ services.
Initial population is obtained by creating specific number of these chromosomes.

\section{- Service Set Creator (SSC)}

SSC algorithm transforms a chromosome to service set in the following steps:

1. For Each gene in chromosome which is in the formula $\left(S_{j}, A_{k}\right)$ do the below step.

1.1. Place $\mathrm{k}^{\text {th }}$ activity in $\mathrm{j}^{\text {th }}$ service as an operation.

\section{- Selection}

Since different attributes of a service conflict with each other, there might not be a unique answer which is optimal considering as attributes. Therefore, we formulate the service identification problem as a multi-objective optimization problem and we look for alternative solutions which are superior to other solutions. These solutions are known as Pareto-optimal solutions or non-dominated solutions [14]. After obtaining these solution sets, architect decides which one of them suit enterprise business goals.

NSGA [14] algorithm is one of the approaches that are used to find Pareto-optimal set. The only difference between this algorithm and a typical GA is the selection operator. In this paper, we adopt the selection operator introduced in NSGA.

\section{- Crossover Operator}

One of the unique and important aspects of the techniques involving $\mathrm{GA}$ is the important role that recombination (traditionally, in the form of crossover operator) plays. In this paper, we propose a new crossover operator, called Service-Crossover. An offspring $\mathrm{C} 1$ is created from two parents, $\mathrm{P} 1$ and $\mathrm{P} 2$ as follows:

1. Given a business process with $\mathrm{n}$ activities, randomly select a number $i,($ where $i=1,2 \ldots n-1)$.

2. Randomly select $i$ activities which are the activities to be passed on from parent to offspring per parent.

3. One gene is copied directly from $\mathrm{P} 1$ to $\mathrm{C} 1$ if it contains one of previously selected $i$ activities.

4. Remained genes are copied from P2 to $\mathrm{C} 1$ if it does not contain any of previously selected i activities.

\section{- Mutation}

After cross-over, chromosomes are mutated. Mutation prevents the algorithm from being trapped in a local optimal. Mutation plays the role of retrieving lost genetic information by randomly distributing genetic information. In this algorithm, to perform mutation operator, two services, $\mathrm{Si}$ and $\mathrm{S}_{\mathrm{j}}$, are selected randomly. Then two activities, $\mathrm{A}_{\mathrm{i}}$ and $\mathrm{A}_{\mathrm{j}}$, which are in $S_{i}$ and $S_{j}$ services respectively, are selected to exchange.

GA is executed as many as the number of generationspan and optimal service set is obtained through this evolutionary process.

\section{Evaluation OF THE Method}

In this section, using a real-world example, we will explain the proposed method. We make use of a set of 
business goals and a business process and then we apply the steps mentioned in section 5 on this particular example to achieve a set of optimal services.

\section{- Metric Definition Phase}

We consider three business goals as inputs in this case study: Agility, Ease of Future Enhancement and High Value to Business.

A set of design metrics which satisfy business goals should be selected or designed in the first phase. According to the fact that many work has been done in the field of defining design metrics [22] we have chosen four design metrics. These metrics are: cohesion, coupling, business entity convergence, and granularity. Considering the mentioned business goals, quality attributes which cover these goals and also the relationship between these metrics and quality attributes are shown in Table I.

TABLE I. RELATIONSHIP BETWEEN BUSINESS GOAL, QUALITY ATTRIBUTE AND METRIC

\begin{tabular}{|c|c|c|}
\hline Business Goal & Quality Attribute & Metric \\
\hline \multirow{2}{*}{ Agility } & Coupling & $\mathrm{V}_{\mathrm{COPL}}$ \\
\cline { 2 - 3 } & Granularity & $\mathrm{V}_{\mathrm{GRANU}}$ \\
\hline Ease of Future Enhancement & Cohesion & $\mathrm{V}_{\mathrm{CHOS}}$ \\
\hline High Value to Business & Entity convergence & $\mathrm{V}_{\mathrm{CONVE}}$ \\
\hline
\end{tabular}

As shown in Table I, agility, ease of future enhancement and high value to business are considered as business goals. Coupling and granularity quality attributes are considered to cover agility [20], cohesion is considered to cover ease of future enhancement and also the business entity convergence is considered to cover high value to business goals [7]. The above quality attributes and their corresponding metrics are adopted from [7].

The selection of above metrics is based on the fact that they are easily measurable, thus suitable to be used in automated service identification. Secondly, these metrics have been defined to measure service attributes in design level. Identifying high quality services in design level will have a great impact on quality of the final product. Also designing the right services in early stages of SOA life cycle provides reduction of costs and errors in the next stages. In previous methods, designer should utilize his experience to decompose business processes. They decomposed processes considering a set of general principles, for example identified services should meet loose coupling and high cohesion. Utilization of design metrics makes quantitative and objective evaluation of the identified services possible. As a result, these metrics can be substituted with designers' subjective comprehension and this completely satisfies the objective of automation in this paper. Thirdly, these metrics are measurable based on information which exists in business processes of an enterprise, like data flow between activities. Finally, these metrics measure quality attributes which cover considered business goals in this paper. Regarding the above reasons, the following metrics were selected.

\section{A. Service Granularity}

Service granularity is a functional scope that each service opposes to environment. Therefore, service granularity is defined as an average of business activities that a service does.

\section{B. Service Coupling}

Service coupling shows how much a service has dependency to other services. Since business process Cerography is performed by calling services according to business process control flow, it is possible that input of a service is obtained from output of other services, and these results in coupling of two services. A request to a service is implemented through a message which is sent to service operations. Size of message indicates the coupling degree which is dependent on number and complexity of informational entities in message.

\section{Service Cohesion}

Service cohesion refers to degree of relation between activities inside a service. Therefore the degree of cohesion depends on two factors: First, the number of activities inside service and second, dataflow between activities.

\section{Business Entity Convergence}

From the information-centric viewpoint, a business process is also a process comprising activities acting on business entities to walk through their lifecycles 7]. The business entities provide a natural way to do a partitioning of business activities into services, where each service is identified to carry out the activities processing the same business entities. Therefore, service set must satisfy two factors: Firstly, one service operates in minimum number of possible business entities. Secondly, activities which operate on the same entities had better be placed in the same service. The formulas for the above metrics can be found in [7].

\section{- Service Sets Identification Phase}

The metrics $\mathrm{V}_{\mathrm{COPL}}, \mathrm{V}_{\mathrm{GRANU}}, \mathrm{V}_{\mathrm{CHOS}}$ and $\mathrm{V}_{\mathrm{CONVE}}$, which had been defined in the first phase, constitute the first input of this phase. Two other inputs of this phase are business processes and business entity weights. We adopt a scenario that is described in [13]. Figure 3 illustrates process model of this scenario.

The particular process model described above is defined as a directed graph delineated by the formal representation in section 4. This specific process model enters as an XML file and in the form of a matrix. The rows and columns of this matrix indicate activities existing in the process. Each element $\mathrm{i}, \mathrm{j}$ of this matrix is the set of business entities which are sent from $i$ th activity to $j$ th activity.

The other task of this phase is modeling of service identification problem based on GA parameters. We have implemented the algorithm to solve the multi-objective problem using C\# language. The developed tool has such a design that takes specific process model in the format of an XML file. Also the ability to set the parameters of GA has been provided.

Parameters of GA were determined as follows for fast convergence: Population size $=500$, Generation $\operatorname{span}=100$, Crossover rate $=0.95$, Probability of mutation $=0.05$ 
One of the chromosomes of the above business process in population is as follows:

(S1, Notify Claim)(S2, Record Claim)(S1, Validate Claim)(S3, Analyze Claim)(S1, Decide on Claim)(S2, Reject claim)(S3, Provide Addition Data)(S4, Review Claim rejection)(S3, Offer Benefit)(S4, Prepare Claim Discharge)(S3, Record Benefit Payment)(S2, Close Claim)(S1, Operate Outgoing Payment).

As above, 500 chromosomes are created and algorithm enters an evolutionary process and finally produces a solution as Pareto solution set.

Non-dominant solution

$A 1=$ Notifyclaim, $A 2=$ Review claim rejection, $A 3=0$ ffer benefit, $A 4=$ Prepare Claim, $A 5=$ Recordclain $A 6=$ Record benefitpayment,$A 7=$ Validate claim, $A 8=$ Analyze claim, $A 9=$ Decide on clain

A10 $=$ Reject claim, $A 11=$ Close claim,$A 12=O$ perate outgoing payment, $A 13=$ Provide additional data

$(\mathrm{S} 1, \mathrm{Al})(\mathrm{S} 2, \mathrm{~A} 2)(\mathrm{S} 2, \mathrm{~A} 3)(\mathrm{S} 2, \mathrm{~A} 4)(\mathrm{S} 1, \mathrm{~A} 5)(\mathrm{S} 2, \mathrm{~A} 6)(\mathrm{S} 1, \mathrm{~A} 7)(\mathrm{Sl}, \mathrm{A} 8)(\mathrm{S} 1, \mathrm{~A} 9)(\mathrm{S} 1, \mathrm{~A} 10)(\mathrm{S} 2, \mathrm{All})(\mathrm{S} 2, \mathrm{Al} 2)(\mathrm{S} 1, \mathrm{Al} 3)$

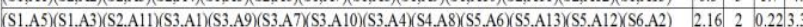

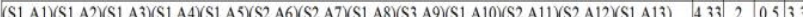

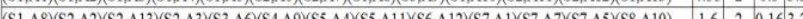

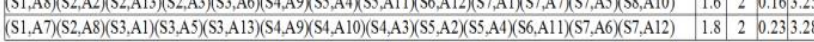

Figure 4. Some results of the second phase

Five Pareto solutions have been shown in Figure 5. Genes inside chromosomes are in the format of (Service, Activity). Moreover, the average value of each metric has been shown for the Pareto sets.

\section{- Appropriate Service Set Selection Phase}

As stated before, the main focus of this paper is on the second phase. Therefore defining proper fuzzy functions and ranking Pareto solutions using fuzzy logic have been considered as future work. In this paper, based on the architect's experience, we have selected one of the Pareto solutions which satisfy the business goals. The most important business goal was Agility, from the authors' point of view. Therefore, we considered Granularity and Loose Coupling quality attributes with the highest priority in our selection. Then, we applied the SSC algorithm to the selected solution. This algorithm maps a solution to a service set. Moreover, the service identification task is carried out by this algorithm. These specifications are obtained based on input and outputs of activities. Business entities existing in each ith row of this matrix form the output of ith activity and business entities in ith column form the input of ith activity. The identified services and their specification have been shown in Figure 5 and Table II respectively.

\begin{tabular}{|l|l|}
\hline \multicolumn{1}{|c|}{ Service 1 } & \multicolumn{1}{c|}{ Service 2 } \\
\hline OP1: Notify Claim & OP1: Review Claim rejection \\
OP2: Record Claim & OP2: Offer Benefit \\
OP3: Validate Claim & OP3: Prepare Claim Discharge \\
OP4: Analyze Claim & OP4: Record Benefit Payment \\
OP5: Decide on Claim & OP5: Close Claim \\
OP6: Reject claim & OP6: Operate Outgoing \\
OP7: Provide Addition Data & Payment \\
\hline
\end{tabular}

Figure 5. Operations of services
TABLE II. THE INTERFACE OF SERVICE 1

\begin{tabular}{|l|c|c|c|}
\hline $\boldsymbol{O p}$ & Input & Output & Corresponding Activity \\
\hline $\boldsymbol{O p} \mathbf{1}$ & - & Loose event ,Claim & Notify Claim \\
\hline $\boldsymbol{O p} \mathbf{2}$ & $\begin{array}{c}\text { Loose event } \\
\text { Claim }\end{array}$ & Claim & Record Claim \\
\hline $\boldsymbol{O p} 3$ & Claim & Claim & Validate Claim \\
\hline $\boldsymbol{O p} 4$ & Claim & - & Analyze Claim \\
\hline $\boldsymbol{O p} 5$ & Claim & Claim, benefit in claim & Decide on Claim \\
\hline $\boldsymbol{O p}$ 6 & Claim & Claim & Reject claim \\
\hline $\boldsymbol{O p} 7$ & Claim & Loose event ,Claim & Provide Addition Data \\
\hline
\end{tabular}

Finally, this service set are exposed as output. In this section we described effectiveness of the proposed approach in a step by step example. Identified services have right level of abstraction. In other words operations existing in services completely focus on one single business functionality. For example, service 1 performs operations related to Claim. Moreover, services are completely in the right status from technical metrics point of view. The most important quality attribute was Granularity, from authors' point of view, which identification of two services from this business process indicates the importance of this attribute. If this method was not applied, the architect would have to identify various service schemas and then compare them manually. This completely depends on the architect's experience. Moreover, since the problem of service identification is considered as NP-complete problem [9], creating all of the schemas is impossible. As we stated in the beginning of this section, a real-world business process has been used in this case study. This indicates that our proposed method could have many applications in enterprises. The most important advantage of this method is that identified services are aligned with the business goals. Therefore, enterprise service-oriented systems can be easily adapted to the changing environments of business. Another advantage of this method is using enterprise-level assets. In this section, we only used business process, an artifact which is available to service identification activities. Moreover, this case study has clearly shown the simplicity of the proposed method. The only activities that architect should conduct are giving business processes to a tool, defining appropriate metrics and receiving a set of optimal services as output.

\section{CONCLUSION AND FUTURE WORK}

In this paper, we proposed a method which automatically identifies enterprise-level services. First, a set of design metrics is defined using business goals. Then by defining a multi-objective GA, a set of non-dominated solutions are selected from alternative solutions. Since this solution has different characteristics, a set of fuzzy rule can be defined to select solutions which satisfy enterprise business goals. Finally, the best solution which is a set of optimal services is derived as output. Automation of service identification helps architects deal with activities which require human inference, such as defining appropriate metrics which satisfy enterprise business goals. This paper focuses mainly on the second phase of the proposed method that is service identification using GA. Therefore, defining proper membership functions and applying fuzzy logic can be considered as future work. 


\section{ACKNOWLEDGMENT}

The project has been partially supported by Shahid Beheshti University under the supervision of Automated Software Engineering Research (ASER) Group, Faculty of Electrical and Computer Engineering. This work was also supported, in part, by Science Foundation Ireland grant 03/CE2/I303_1 to Lero - the Irish Software Engineering Research Centre (www.lero.ie)

\section{REFERENCES}

[1] M. Kim, S. Kim, "Service Identification Using Goal and Scenario in Service Oriented Architecture," 15th Asia-Pacific Software Engineering Conference, IEEE, 2008, pp. 419-426.

[2] L. Ackerman, A. D. Bari, G. Hodgkinson, A. Kesterton, L. Olson, B. Portier, U. Wahli, "Building SOA Solutions Using the Rational SDP," IBM Corp, 2007.

[3] T. Erl, "Service-Oriented Architecture Concepts, Technology, and Design," Prentice Hall PTR, 2005.

[4] A. Arsanjani, "Service-Oriented Modeling and Architecture (SOMA)," IBM developer Works, 2004.

[5] S. Ghosh, A. Allam, T. Abdollah, S. Ganapathy, K. Holley, A Arsanjani, "SOMA: A method for developing service-oriented solutions," IBM System Journal, vol. 47, 2008.

[6] G. K. Behara, S. Inaganti, S, "Service Identification: BPM and SOA Handshake," Business Process Trends, 2007.

[7] N. Zhou, Y. Zhu, Y, H. Wang, Q. Ma, "Evaluating Service Identification with Design Metrics on Business Process Decomposition," International Conference on Services Computing, IEEE, 2009.

[8] P. Jamshidi, M. Sharifi, S. Mansour, "To Establish Enterprise Service Model from Enterprise Business Model," Proc. of IEEE International Conference on Services Computing, 2008.

[9] H. Jain, H. Zhao, N. R. Chinta, "A Spanning Tree Based Approach to Identifying Web Services," International Journal of Web Services Research, 2004

[10] A. Flitman, D. Moody, "A methodology for clustering entity relationship models-a human information processing approach," 18 th international conference on the entity-relationship approach, berlin, 1999, pp. 114-130.

[11] L. G. Azevedo, et al. "A Method for Service Identification from Business Process Models in a SOA Approach," Springer, 2009, pp. $99-112$.

[12] P. Nandi, S. Kumaran, S. Ghosh, A. Arsanjani, J. L. Strosnider, "Model-driven synthesis of SOA solutions", IBM System Journal 47(3), 2008

[13] S. Kumaran, et al, "On the Duality of Information-Centric and Activity-Centric Models of Business Processes," CAiSE, Springer, 2008, pp. 32-47.

[14] G. B. Lamont, V. Veldhuizen, D. A. Coello, "Evolutionary Algorithms for Solving Multi-Objective Problems," Second Edition, Springer, 2007.

[15] U. Apte, C. S. Snaker, "Reusability-based Strategy for Development of Information Systems," MIS Quarterly, 14(4), 1990.

[16] K. Levi, A.arsanjani, "A Goal-driven Approach to Enterprise Component Identification and Specification," Communications of the ACM, 45(10), 2002.

[17] S. Johnston and J. Smith, "RUP Plug-In for SOA V1.0," IBM DeveloperWorks, Available at http://www.ibm.com $\backslash$ developerworks/rational/library/05/510_soaplug I, 2005.

[18] N. Bieberstein, R. G. Laird, K. Jones, T. Mitra, "Executing SOA: a practical guide for the service-oriented architect," IBM Press, 2008.

[19] M. Bell, E. A. Marks, "Executive's Guide to Service-Oriented Architecture. Canada," John Wiley \& Sons, New Jersey, 2006.

[20] L. Bass, P. Merson, L. O'Brien, "Quality Attributes and ServiceOriented Architectures," Department of Defense, Technical Report September, 2005.

[21] R. Veryard, L. Wilkes, "Service-Oriented Architecture: Considerations for Agile Systems," http://msdn.microsoft.com/library/default.asp?url=/library/enus/dnmaj/html/aj2service.asp, 2004.

[22] A. Rostampour, A. Kazemi, F. Shams, A. Zamiri, P. Jamshidi, "A Metric for Measuring the Degree of Entity-Centric Service Cohesion," Service oriented Computing and Application (SOCA), 2010

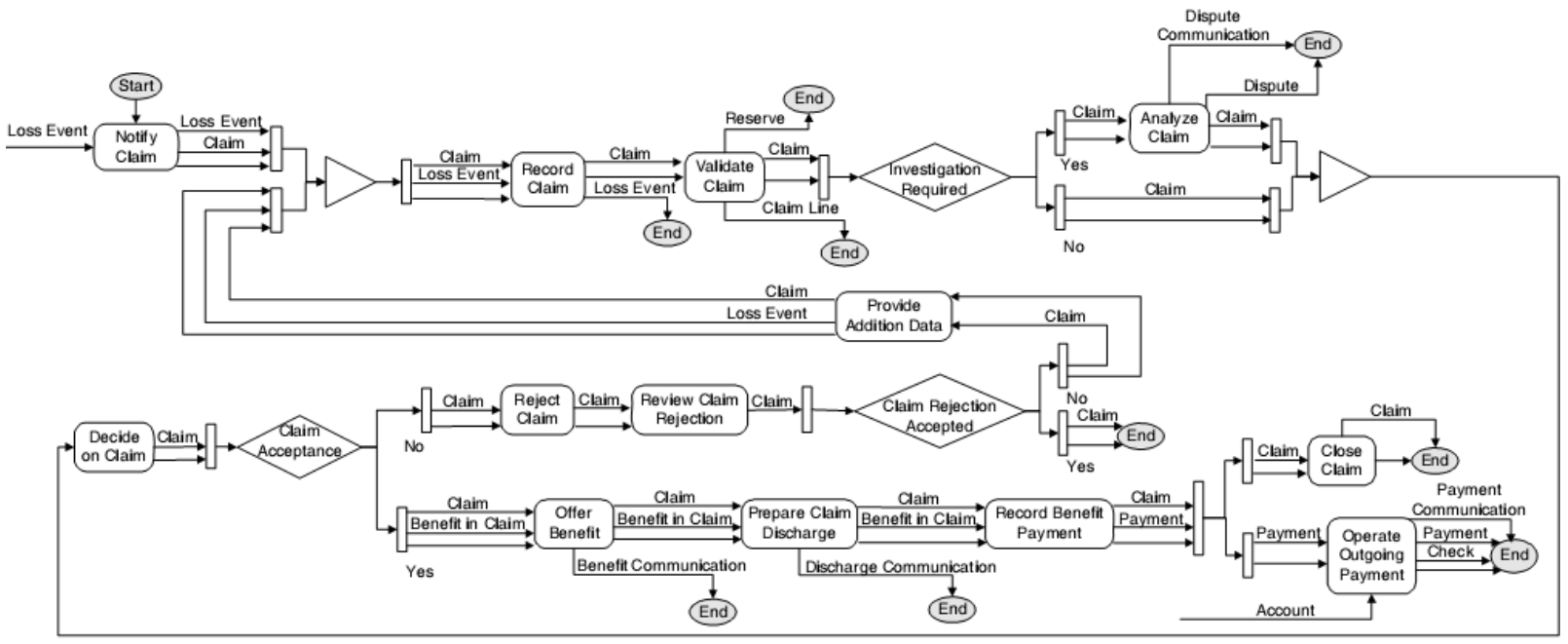

Figure 3. Administering property damage claim [13] 